\title{
The Use of TRIDYN and Medium Energy Ion Scattering to Calibrate an Industrial Arsenic Plasma Doping Process
}

Running title: The Use of TRIDYN and MEIS

Running Authors: England et al

$$
\text { J. Englanda) }
$$

Varian Semiconductor Equipment, Silicon Systems Group, Applied Materials Inc., 35 Dory Road, Gloucester, MA 01930 USA

Surrey Ion Beam Centre, Advanced Technology Institute, University of Surrey, Guildford Surrey GU2 7XH UK

\section{J. A. van den Berg and A. Rossall}

Ion Beam Centre, School of Computing and Engineering, University of Huddersfield, Huddersfield, HD1 3DH, UK.

\section{a) Electronic mail: j.england@surrey.ac.uk}

Plasma doping ion implantation (PLAD) is becoming increasingly important for enabling the manufacture of advanced semiconductor devices. In this study a VIISTA PLAD implanter was used to implant planar $300 \mathrm{~mm}$ Si wafers with As/7keV from an arsine containing plasma with a total ion fluence of $1 \times 10^{16}$ ions $/ \mathrm{cm}^{2}$. The wafers then underwent a wet chemical clean and anneal to mimic a full industrial process flow. The effects of each process step were measured using cross sectional TEM images, TEM/EDS measurements and by Medium Energy Ion Scattering (MEIS). The PLAD implantation process was modelled using TRIDYN, a dynamic, binary collision approximation model that accounted for the interactions between wafers and the ions and neutrals produced by the PLAD implanter. MEIS spectra were analysed to extract elemental concentration depth profiles using POWERMEIS guided by the outputs of the TRIDYN model. The input 
fluxes of the TRIDYN model were calibrated such that the predicted TRIDYN and MEIS profiles were self-consistent. Combining the different analysis techniques and considering elemental concentrations alongside a TRIDYN model enabled magnitudes of ion and neutral fluxes of $\mathrm{Si}, \mathrm{As}$ and $\mathrm{H}$ to be proposed and the relative importance of direct implantation and ion beam mixing during the PLAD implant to be revealed. This in turn led to proposals for the sources of the ion and neutral species, the importance of Si neutrals originating from the plasma chamber over those originating from the Si bulk in the "deposited" layer being of particular interest. Following the evolution of the as-implanted profiles through the wet clean and anneal steps gave insights into how the PLAD implant affected the results of the full process flow.

\section{INTRODUCTION}

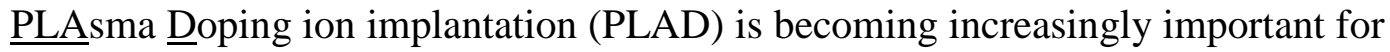
enabling the manufacture of advanced semiconductor devices. Implantation has historically largely been used to dope devices with implants of relatively high energy (less than $10 \mathrm{keV}$, say) and/or low fluence $\left(\sim 1 \times 10^{15} \mathrm{ions} / \mathrm{cm}^{2}\right.$ and below) but implant processes of higher fluence $\left(\sim 1 \times 10^{16}\right.$ ions $/ \mathrm{cm}^{2}$ and above) and lower energy (less than $10 \mathrm{keV}$ and often below $1 \mathrm{keV}$ ) have become common. An approach to maintain productivity in industrial scale manufacturing is to move implant processes from beamline based implanters to PLAD tools. PLAD is simple in concept: a negatively biased substrate immersed in a plasma is doped by ions and neutrals from that plasma. However, a fundamental understanding of PLAD must take into account high fluence implantation, deposition, sputtering and ion beam mixing during the implant after which passivation, cleaning and annealing steps have to be considered. The flux of ions to the wafer can be 
measured, but the composition of the ions is unknown. The fluxes and compositions of neutral species that deposit on the substrate surface during PLAD cannot be directly measured. Neutrals and ions can arise from different sources, notably from the feed gas, deposited layers, chamber components and the processed wafers themselves. In the process investigated in this study, arsenic containing species could contain one or more hydrogen atoms and whilst some hydrogen would be expected to be chemically bonded in material deposited on the wafer, some may escape during or after the implant. This means that PLAD might form thin layers of complex, unusual stoichiometries with variable compositions and densities.

The measurement of PLAD dopant profiles in conjunction with process modelling should allow the compositions and fluxes of the neutral and ion species and a deeper understanding of the PLAD process ${ }^{1}$. However thin, complex layers are difficult to measure. Profiling methods such as Secondary Ion Mass Spectroscopy and Dynamic xray Photoelectron Spectroscopy suffer from profile distortion due to sputter mixing and matrix dependent effects. Quantification of these effects for SIMS measurements of As in Si has been reported before ${ }^{2}$ but is not routinely available to allow SIMS to be useful for this study. Cross sectional images collected in a transmission electron microscope (TEM) were used in this study to measure the thickness of layers and to yield further qualitative information on crystallinity, damage and composition through $\mathrm{Z}$ contrast. In addition, energy dispersive spectroscopy (EDS) in the TEM was used to measure elemental compositions of layers. This technique provided atomic fractions of selected elements in a layer, but not the absolute number of atoms; TEM/EDS could not measure layer densities, which were the primary unknowns in this study. 
Medium Energy Ion Scattering (MEIS) is a technique that avoids some of these pitfalls; MEIS measurements on the samples discussed in this study have been reported elsewhere ${ }^{3,4}$. MEIS is sensitive to the total number of atoms in a layer (atoms $/ \mathrm{cm}^{2}$ ) and in the absence of information from other sources cannot determine layer thicknesses and densities. MEIS spectra are usually analysed with the assumption that the elements detected are present at constant atomic fractions throughout a layer and often as part of compounds of standard stoichiometry and standard density. In this study, the layers were not assumed ab-initio to be uniform or be composed of standard compounds but to have compositions and densities consistent with those predicted from the results of a model of the PLAD process calculated using the dynamic, binary collision approximation code TRIDYN $^{5}$. This study proposes a simple model that the silicon implant profile is unchanged during post implant clean and anneal processes. The validity of this model was tested by comparing post clean and post anneal MEIS measurements with those POWERMEIS ${ }^{6}$ simulations based on TRIDYN model inputs. Consistency was also checked against thickness information from TEM images and atomic fraction profiles from TEM/EDS measurements. Modifications to the as-implanted TRIDYN arsenic profile and the introduction of oxygen (that was not part of the TRIDYN implant model) gave valuable insights into mechanisms acting in the post implant processes.

\section{EXPERIMENTAL}

\section{A. WAFER Processing}

\section{PLAD Implantation}

Bare $300 \mathrm{~mm}$ diameter silicon wafers were implanted with arsenic in a VIISta PLAD system at Applied Materials, Varian Business Unit in Gloucester, MA. The PLAD system is described in more detail in ${ }^{7}$. Briefly, a continuous RF plasma was generated in 
a low pressure gas mixture containing $\mathrm{AsH}_{3}$. The plasma composition could be monitored using an optical emission spectrometer (OES). The wafer, immersed in the plasma, was biased to $-7 \mathrm{kV}$ in a pulsed manner, which determined the energy of ions extracted from the plasma to bombard the wafer. A shield ring surrounded the wafer perimeter to ensure that ion extraction was uniform across plasma. The number of ions extracted from the plasma in each bias pulse was detected in a Faraday detector behind a slit in the shield ring. Because there was no mass analysis, the composition of the ions was unknown. The implants were halted once the total ion fluence had reached $1 \times 10^{16} / \mathrm{cm}^{2}$.

The plasma chamber was pre-conditioned by running a number of sacrificial, nonproduct wafers to coat the chamber walls with predominantly arsenic and silicon. After an implant was completed, the unbiased wafer was exposed to an in-situ plasma based passivation process for a few seconds to clean its surface of excess arsenic that would otherwise be emitted when the wafer was removed from the PLAD chamber and exposed to atmosphere.

\section{Full Process Flow}

Although this study did not involve any pre-implant lithography steps, the wafers underwent an industry standard SPM (a 70\% Sulphuric acid, 5.6\% hydrogen Peroxide, $24.4 \%$ water Mixture) wet chemical clean immediately after the implant to represent the production step of photo-resist removal. The SPM clean was applied at $120^{\circ} \mathrm{C}$ in a temperature controlled bath for 5 minutes in a Nexgen Technologies wet bench. The SPM mixture composition was monitored and adjusted daily. The sulphuric acid removes photoresist and organic contaminants, but is not expected to attack silicon. Hydrogen peroxide is a strong oxidising agent and can be expected to oxidise surface silicon ${ }^{8}$. 
After the wet clean, the wafers were "spike" annealed at $1050^{\circ} \mathrm{C}$ held for $1.7 \mathrm{~s}$ in a nitrogen atmosphere containing 100ppm of oxygen and a dilute hydrofluoric (DHF) acid step could be applied to remove surface oxide.

\section{B. Metrology}

Full wafers were processed and then broken into samples to be sent for analysis. It should be pointed out that measurements of this study were made on samples that were processed at different times over a 27 month period, not from one experiment. The possibility of process variations must be considered when comparing analyses from different samples. Studies of how chamber conditioning affects PLAD process have been carried out and found not to change the results significantly from those presented in this study ${ }^{9}$. Furthermore, samples are normally considered to remain unchanged between the end of the process in which they were formed and the time of analysis; an example of where this is not the case is discussed in the evolution of post implant sample ${ }^{4}$.

\section{TEM and TEM/EDS}

Bright field TEM images were made by commercial analytical laboratories on samples that had been coated with iridium before the TEM lamellae were produced. Energy dispersive spectroscopy (TEM/EDS) measurements were made on two of these samples to determine elemental atomic fraction profiles. The lateral resolution of TEM/EDS may be taken as around 2nm (from the interaction region of electrons used to excite the x-rays). The atomic concentrations measured by MEIS for post implant and post clean samples were converted to atomic fractions and agreed broadly with TEM/EDS measurements of different samples.

\section{MEIS Measurements}


Samples for MEIS measurements required no sample preparation and were measured as soon after processing as soon as international shipping timescales allowed. MEIS measurements, described in more detail in ${ }^{3,4}$ were carried out on the University of Huddersfield MEIS system ${ }^{1,10}$ using $100 \mathrm{keV} \mathrm{He}^{+}$ions and a scattering angle of $90^{\circ}$.

\section{MEIS Spectrum Analysis Method}

In this study, elemental profiles were extracted from the measured MEIS energy spectra using POWERMEIS ${ }^{6}$ which was run from its website (http://tars.if.ufrgs.br/) between May 2016 and September 2017 using the SRIM, Chu and Marion options to calculate stopping powers, energy straggling and beam charge neutralisation corrections respectively. Only single scattering events were accounted for. POWERMEIS took several minutes to simulate a typical spectrum. Each sample substrate was divided into layers defined by their elemental atomic fractions and total densities. Optimisation of the atomic fractions and densities of the layers was carried out manually to produce the best fit of the simulated to measured MEIS spectra, the quality of fit being judged by eye. Model layer thicknesses were normally chosen to be $2 \mathrm{~nm}$ to give enough fidelity to the fits without requiring a large number of layers that would make manual fitting too onerous, although some $1 \mathrm{~nm}$ thick layers were defined in some interface regions. Around ten manual optimisation cycles were sufficient to obtain a pleasing fit. Automated adjustment of the layer compositions to minimise a fitting function based on the comparison between the fit and experimental data, such as chi squared, was not available but would be a valuable future capability. As POWERMEIS is only truly applicable to randomly oriented samples,

a method for analysing aligned spectra was devised in which the elemental profiles used to fit a random spectrum where divided into two parts to fit a corresponding aligned spectrum. 
The first part contributed to electronic energy loss and the measured backscattered signals from non-crystalline surface regions of the samples and interstitial atoms, whilst the second part only described the energy loss and scattering from the crystalline regions. This is similar to the approach summarised in ${ }^{11}$. Comparison of the aligned and random spectra allowed the amounts of substitutional and interstitial As and Si damage profiles to be estimated.

\section{Guiding MEIS analysis using TRIDYN}

As explained in the introduction, fitting a MEIS spectrum determines atomic areal densities (atoms $/ \mathrm{cm}^{2}$ ) but in isolation cannot always generate a single, unique determination of elemental profiles (atoms $/ \mathrm{cm}^{3} / \mathrm{nm}$ ). TEM cross sectional measurements and TRIDYN model profiles were combined to generate self-consistent results with POWERMEIS in the following way:

1. Trial ion and neutral particle fluxes were input into a TRIDYN model to calculate post implant As, Si and $\mathrm{H}$ atoms concentration profiles (atoms $/ \mathrm{cm}^{3}$ vs depth);

2. The TRIDYN model concentration profiles were used to derive the layer properties (atomic fractions and total densities) to be used by POWERMEIS to simulate the expected MEIS energy spectrum (counts/channel vs energy);

3. The POWERMEIS input layer values were modified to optimise the agreement between the simulated and the measured MEIS spectra;

4. The optimised POWERMEIS solution was plotted as elemental atomic concentration profiles (atoms $/ \mathrm{cm}^{3}$ vs depth);

5. If the TRIDYN and POWERMEIS profiles were not in good agreement, a new TRIDYN model was generated by modifying the input particle fluxes. 
This cycle was repeated until the TRIDYN and optimum POWERMEIS atomic profiles matched, the quality of fit again being judged by eye. The predicted as implanted depth profiles had to be consistent with layer thicknesses measured in TEM cross sections. The assumptions to the TRIDYN model were kept simple to avoid overfitting the model with too many parameters. In particular, ion and neutral fluxes were held constant throughout the PLAD implant.

The Si profile generated by the TRIDYN implant model was then used as the basis for POWERMEIS simulations of MEIS spectra measured after wet clean and post anneal. The assumption that the Si profile remained the same for the post-implant, post clean and post anneal samples means that effects such as redistribution of the profiles by the wet chemistry have been ignored. Discrepancies between the TRIDYN and POWERMEIS profiles were expected to show whether the constant input flux input and Si profile assumptions could be improved upon.

\section{TRIDYN Model}

SRIM (Stopping Range in Materials) and its component TRIM (TRansport of Ions in Matter) can be considered a reference model for simulating the range of energetic particles injected into amorphous materials ${ }^{12}$. TRIM uses a binary collision approximation (BCA) to model the collision cascades of particles injected into a substrate that is described by layers of defined composition and thickness which are not altered (i.e. are held "static") throughout the calculation. The high fluences appropriate to PLAD implants means that the substrate changes greatly, so that a static model is not appropriate to describe such

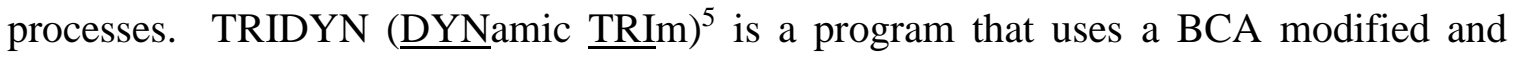


extended from an early version of TRIM to calculate collisional cascades. As in TRIM, the initial substrate is described using homogeneous layers of initial composition and thicknesses but a major difference is that TRIDYN then alters the substrate throughout the calculation to account for the injected particles, sputtered atoms and ion beam mixing. The detailed assumptions used in the BCA have been shown to affect modelling results ${ }^{13}$. The assumptions used TRIDYN's BCA and substrate relaxation algorithms are described in detail in the its manual ${ }^{14}$.

TRIDYN models in this study used a starting substrate described by 200 layers, each $1 \mathrm{~nm}$ thick, a dimension chosen to represent the elemental profiles with sufficient fidelity and exceed the mean free path length between collisions (a necessary condition for valid TRIDYN calculations ${ }^{14}$ ). Oxygen was not included in the model as any surface native oxide layer on the wafer was assumed to be quickly stripped by the chemical action of hydrogen in the plasma ${ }^{15}$. The models used standard parameters for atomic volumes calculated from the densities of common compounds. Surface binding energies (SBE) were mostly calculated from the standard enthalpies of elements and compounds as distributed with the code except for the values of the SBE for As-As, Si-H and As-H which were taken to be $3.1,10$ and $10 \mathrm{eV}$ respectively. Internal displacement energies (Ed) were assumed to be $8 \mathrm{eV}$ for all atoms. Collision cascades were followed for each injected particle and recoiled substrate atom until their energies dropped below $3 \mathrm{eV}$. Ions were given initial kinetic energies of $7 \mathrm{keV}$. The kinetic energies of the deposited neutral species were taken as zero. A model that launched particles from a remote position towards a surface would require particles to have kinetic energy to travel to be deposited on that surface. TRIDYN started by considering particles that had arrived at the surface and so 
did not require the particle to have any kinetic energy. In reality, neutrals arriving at the substrate surface would have possessed at least thermal energy, but since this was below the $3 \mathrm{eV}$ cut off used in the TRIDYN model there was no need to account for this. Higher energy neutral species that could have contributed to collision cascades were not included in this simple model. The magnitudes of the particle fluxes considered in the TRIDYN model were varied to fit the measured elemental profiles. These fluxes, all assumed to have normal incidence and no angular divergence, are summarised in Table 1.

Table 1. Fluxes considered in the TRIDYN model.

Type Description

$\mathrm{As}^{+} \quad$ Generated from the plasma feed gas and from material sputtered from the wafer or chamber walls (either as charged particles or as neutrals which are subsequently ionised in the plasma). Multiply charged ions, As dimers and $\mathrm{H}$ associated with the charged As species have not been included in the TRIDYN model.

$\mathrm{Si}^{+} \quad$ These can be formed by ionisation in the plasma of Si sputtered from the wafer and chamber walls or liberated by chemical etching of quartz or $\mathrm{SiO}_{2}$ layers by $\mathrm{H}$ radicals. This flux is required in the TRIDYN model.

$\mathrm{H}+\quad \mathrm{H}$ monomer, dimer and trimer peaks are routinely observed by residual gas analysers ${ }^{16}$. Energetic hydrogen ions formed from the $\mathrm{H}$ feed gas and even $\mathrm{H}$ introduced as part of $\mathrm{AsH}_{\mathrm{x}}{ }^{+}$ions deeply penetrate the sample beyond the region 
of interest in this study. For this reason $\mathrm{H}+$ ions have not been included in the TRIDYN model.

$\mathrm{As}^{0} \quad$ Generated from the plasma feed gas and from material sputtered from the wafer or chamber. Some of these neutrals represent $\mathrm{AsH}_{\mathrm{x}}$ molecules that contain arsenic and one or more hydrogen atoms.

$\mathrm{Si}^{0} \quad$ Sputtered from the wafer and the chamber.

$\mathrm{H}^{0} \quad$ Unlike the ions, hydrogen introduced as members of $\mathrm{AsH}_{\mathrm{x}}$ molecules will remain in the deposited layer of interest for this study. The volume taken up by this $\mathrm{H}$ will account for the As not taking up the full density of elemental As.

The inductively coupled plasma in the PLAD system was run continuously so that the breakup of feed gas in the plasma to produce neutrals and radicals (and thereby deposition on the wafer) was also continuous. Ions were extracted across the plasma sheath when the wafer was biased, so the high energy ions arrived in pulses. As and Si ions were assumed to be half the total measured ion flux, with the balance being atomic and molecular hydrogen ions (which were excluded from the model). If ion sputtering directed neutrals onto the wafer, or ion assisted deposition occurred, some of the deposition may have been pulsed, too. Considering only species from the plasma, using simple gas kinetic theory, it could be estimated that a typical "sticking coefficient" of a neutral bombarding a wafer surface was approximately $0.6 \%$ and that a typical ratio of the flux of ions to neutral atoms that stick to the wafer ions was expected to be around 1:10. It might be thought that the pulsed nature of the ion arrivals (but continuous arrival of neutrals) would require a method of running TRIDYN to emulate this pulsed mode. However, the number of neutrals 
deposited between ion pulses was much less than a monolayer and therefore it was justified to run TRIDYN in a conventional way, assuming continuous arrivals of ions and neutrals.

The fluxes in the TRIDYN model were taken to be constant through the implant because this was the simplest assumption to start with. This is known to be incorrect. OES spectra show that the plasma composition varies throughout the implant and the deposition on the chamber walls originating from material sputtered from the wafer surface is also expected to vary as the state of the wafer surface changes. At the beginning of the implant $\mathrm{Si}$ and $\mathrm{SiO}_{2}$ are sputtered from the fresh wafer and can deposit on the walls or be trapped in plasma, available to be recycled onto the wafer. As the implant progresses, the wafer surface contains more As; the amount of As sputtered will increase and can potentially be recycled from the plasma or chamber wall back onto the wafer.

The role of the hydrogen in the present TRIDYN model needs further explanation. Due to the mass difference between $\mathrm{H}$ atoms and the other, much heavier energetic particles, TRIDYN predicts that a bound $\mathrm{H}$ cannot receive sufficient energy during a nuclear collision to escape from the substrate. In another system involving carbon, a sputter mechanism for hydrogen by ion induced bond breaking was proposed ${ }^{17}$, but this mechanism is not included in the TRIDYN model of the present study. The inward flux of hydrogen in the present TRIDYN model is the aggregate of the true inward flux and subsequent sputter losses. The concentration and volume occupied by the hydrogen are used in the TRIDYN model to represent any under-density of the As rich deposited film. After choosing to use the standard atomic density for hydrogen suggested by TRIDYN, the H flux was set to make the TRIDYN results consistent with the as-implanted MEIS spectra. If we had chosen a different value for the $\mathrm{H}$ atomic density, we would have had to set a 
different value for the $\mathrm{H}$ flux. Therefore the reported hydrogen concentrations in the deposited layer are not necessarily a representation of the true concentration of hydrogen in the layer. Measuring the hydrogen distribution in the study was not possible because hydrogen x-rays are too low in energy to be detected by TEM/EDS. In MEIS measurements, the cross section for ion scattering of $\mathrm{He}$ from $\mathrm{H}$ is low, and the scattered He ion energy is low, so that the hydrogen scattering events in the MEIS spectra cannot be differentiated from the high background of scattering events involving Si substrate atoms. Hydrogen could have been measured by SIMS but this measurement would have the unknown matrix effects discussed in the introduction. Elastic recoil detection analysis could be used in the future to determine the hydrogen profile as initial ERD measurements of the hydrogen profile of a similar PLAD process have been made ${ }^{18}$.

\section{Results and Interpretations}

\section{A. Initial Observations of Process Steps}

The four TEM cross sections produced after implant, clean, anneal and oxide removal steps are shown in Figure 1. In all the TEM images in this paper, the wafer surfaces have been oriented vertically so that the ions and neutrals during the PLAD implant arrived in a horizontal direction from the left hand side of the image. The first dark layer on the left of all the images is the iridium cap deposited when making the TEM lamellae. The alignment of the images in Figure 1 suggests that the following processes occurred: 

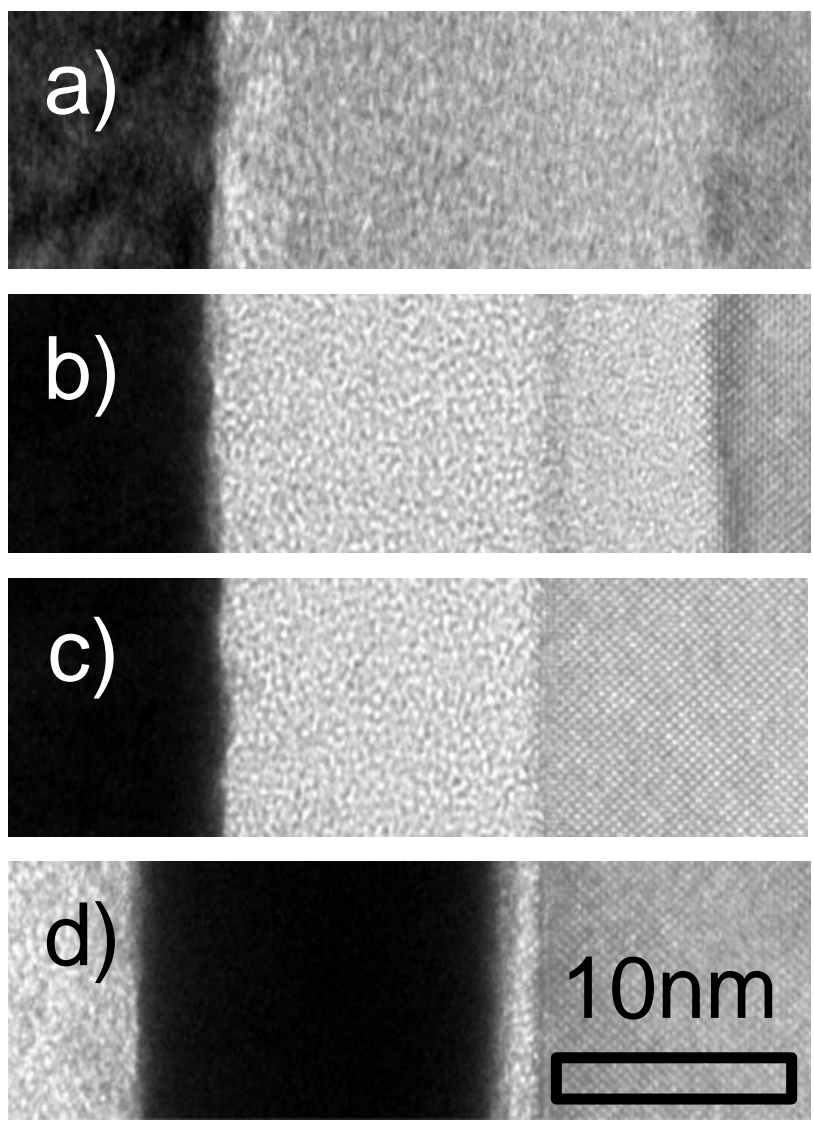

Figure 1. TEM cross sections of samples from different process steps a) several days after implant; b) after an SPM clean; c) post anneal; d) after DHF oxide removal. All the cross sections are shown at the same scale.

During the implant, an amorphous layer approximately 20nm deep was formed beneath the surface. The amorphous layer appears to be uniform in its greyscale until just beneath the surface, where a light band shows the formation of an oxide, presumably by the post passivation treatment and subsequent oxidation during storage in atmosphere.

During the clean, the uniform post implant amorphous layer became divided into upper and lower layers separated by a dark band. During the anneal, the lower layer was re-crystallised up to the dark band (which suggests that the lower layer was amorphous 
silicon); the upper layer above the dark band appeared unchanged. The DHF removed this upper layer which suggests that it was silicon oxide. A light surface layer remained after the DHF that could be native oxide that has re-grown on the cleaned surface. The anneal also appears to have removed blotches in the crystalline $\mathrm{Si}$ substrate which are characteristic of damage from $\mathrm{H}$ implantation.

\section{B. Post PLAD Implant}

\section{TEM/EDS}

Figure 2 shows a cross sectional TEM image of a post implanted planar wafer with EDS measurements superimposed. An amorphous/crystalline interface at $+20 \mathrm{~nm}$ had been created, deeper than the depth at which EDS had detected the presence of As. A light coloured 2.9nm thick surface layer indicates a region from which As had effused after the implant, leaving behind Si which had then been oxidised. There appears to be no buried $\mathrm{O}$, validating the assumption that $\mathrm{H}$ from the plasma had rapidly removed native oxide from the wafer. The presence of Ir beneath the sample surface implies that the TEM lamella preparation steps had disrupted the sample surface. 


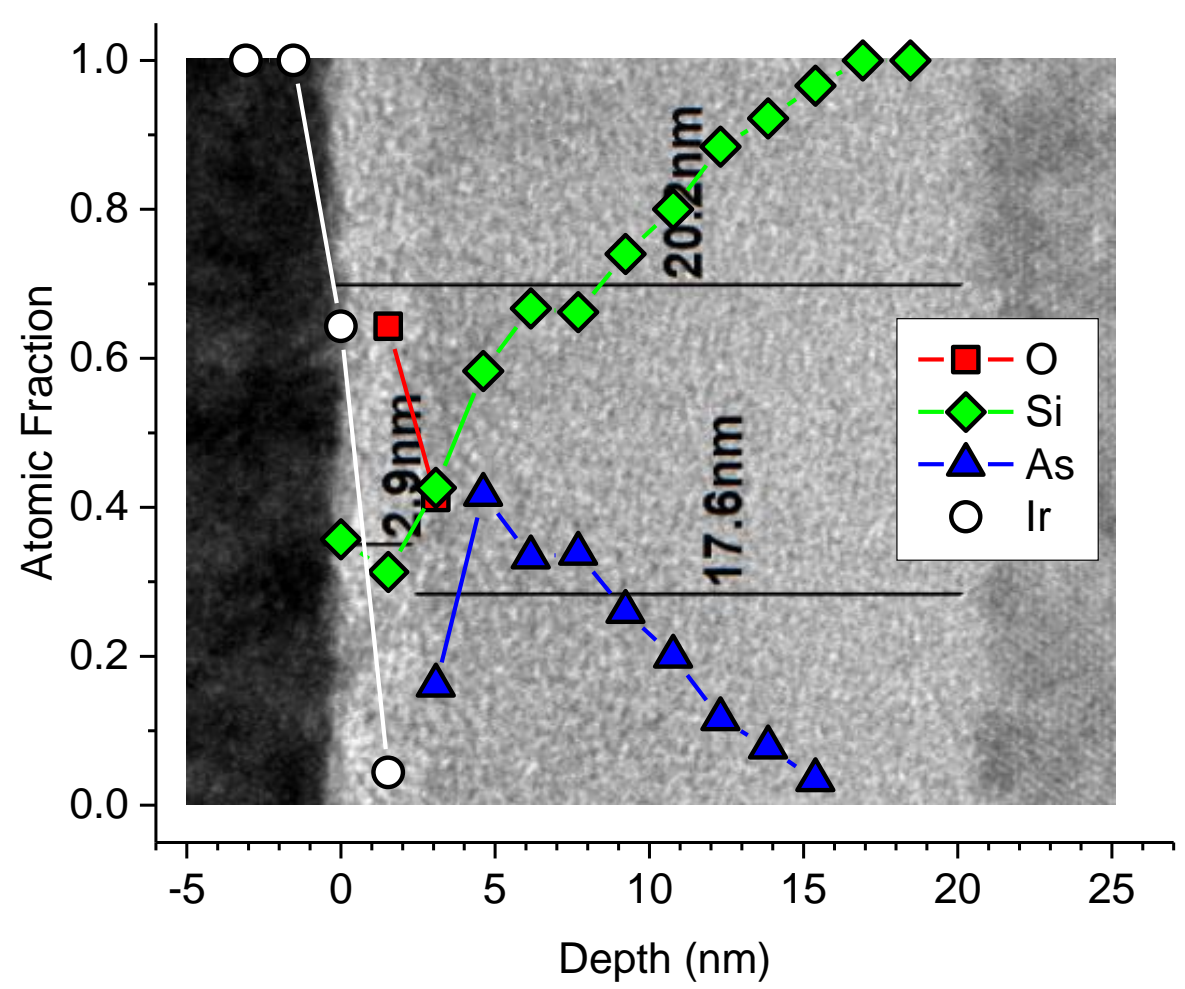

Figure 2. TEM cross section of a post implant sample with EDS measurements superimposed. The origin for the depth axis taken as the bottom of the iridium cap.

\section{MEIS and TRIDYN Model Predictions}

MEIS measurements on a post implanted sample are described and discussed in detail in a companion paper ${ }^{4}$. The MEIS measurements were made on a different wafer than that used in Figure 2. Figure 3 shows profiles extracted from the MEIS spectra for a measurement made as soon as possible after the implant was completed (which was several days due to wafer transport between Gloucester, MA and Huddersfield). The As profile measured at the surface of the deposited layer (between -12 to $-8 \mathrm{~nm}$ ) is not in good agreement with the TRIDYN model because the later does not include the effect of oxidation by the post implant oxygen plasma treatment and exposure to atmosphere. A comparison between the measured and TRIDYN model profiles suggest that As is not only 
lost from the surface but is also "snow-ploughed" into the substrate by the oxidation $\operatorname{process}^{19}$.

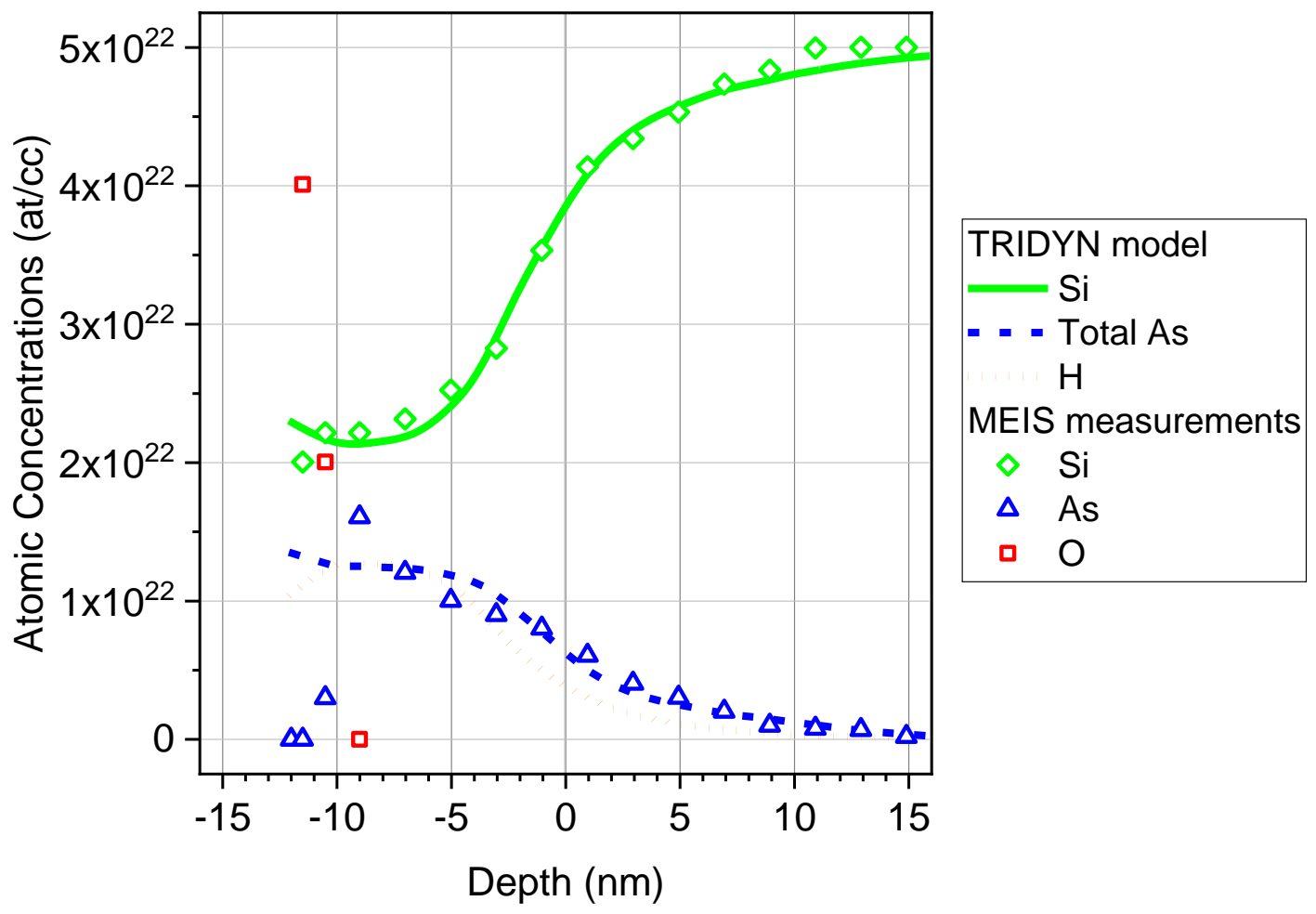

Figure 3. Elemental profiles (symbols) for the post implanted sample extracted from the post implant MEIS spectra using POWERMEIS. The lines show predictions for the $\mathrm{Si}, \mathrm{As}$ and $\mathrm{H}$ profiles from the TRIDYN model. The oxygen was introduced after the implant and is not part of the TRIDYN model; $\mathrm{H}$ cannot be measured by MEIS. The origin for the depth axis has been taken as the original surface of the wafer prior to implantation in the TRIDYN model.

Figure 4 shows the TRIDYN model in more detail, in particular showing the contribution of the As ions and neutrals, Si ions and neutrals and Si from the bulk. The As profile up to $\sim 5 \mathrm{~nm}$ below the original substrate surface was dominated by deposited As 
neutrals that had been recoil implanted by As and Si ions. The As profile deeper than $5 \mathrm{~nm}$ was dominated by As ions, which also contributed to the damage to the Si substrate atoms shown in Figure 4. Comparison to the position of the amorphous/crystalline interface in Figure 2 suggests that $\mathrm{Si}$ displacements need to exceed $\sim 7$ per atom for the cumulative damage to lead to amorphisation.

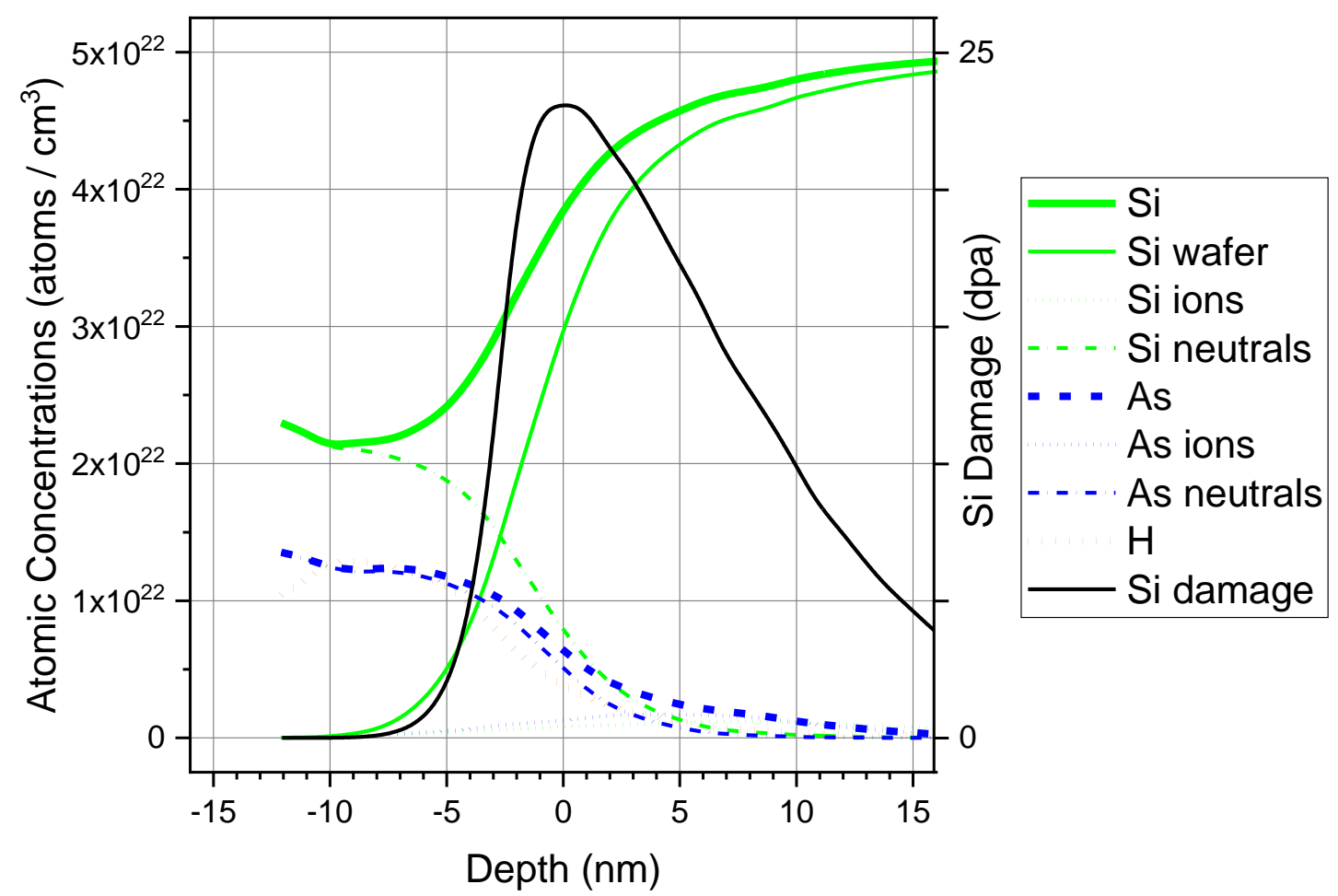

Figure 4. Elemental depth profiles and the contributions from ion and neutral species for the post implanted sample predicted by the TRIDYN model. The damage to the bulk $\mathrm{Si}$ is expressed as displacements per atom.

The best fit TRIDYN model (Figures 3 and 4)), assumed that the ion fluences for the $1 \times 10^{16} / \mathrm{cm}^{2}$ process were $25 \%$ silicon, $25 \%$ arsenic and $50 \%$ hydrogen. The total 
neutral fluences were $6.2 \times 10^{16} / \mathrm{cm}^{2}$ composed of $47 \%$ silicon, $27 \%$ arsenic and $26 \%$ hydrogen atoms. The effects of changing the $\mathrm{Si}$ and As neutral fluences in the TRIDYN model are shown in Figure 5. The TRIDYN model assuming no external Si suggests that the amount of Si measured to be present in the intermixed layer could not have been due only to Si that had been ion beam mixed in from the substrate; additional external fluxes of $\mathrm{Si}$ atoms and ions were necessary. It should also be noted that the fluence of silicon neutrals was higher than that of the arsenic neutrals. Because there was no Si containing gas fed into the plasma, this implies that $\mathrm{Si}$ from the chamber was an important component in the PLAD process. The Si containing ring around the wafer, which is heated by the implant process and thereby does not become coated with deposited arsenic, could be a primary source of this silicon, whilst other sources could be through chemical etching (by the hydrogen) of silicon dioxide parts or sputtering of silicon coated chamber components. The conclusion is not quite the same for the As because the arsine fed into the plasma must have been an important contributor to the total As fluence. TRIDYN calculates the total number of particles sputtered from the wafer surface over the whole implant to be $4.7 \times 10^{15} / \mathrm{cm}^{2}$ for silicon and $1.8 \times 10^{15} / \mathrm{cm}^{2}$ for arsenic, indicating that the incoming Si and 
As could not have been be delivered by recycling material sputtered from the wafer itself.

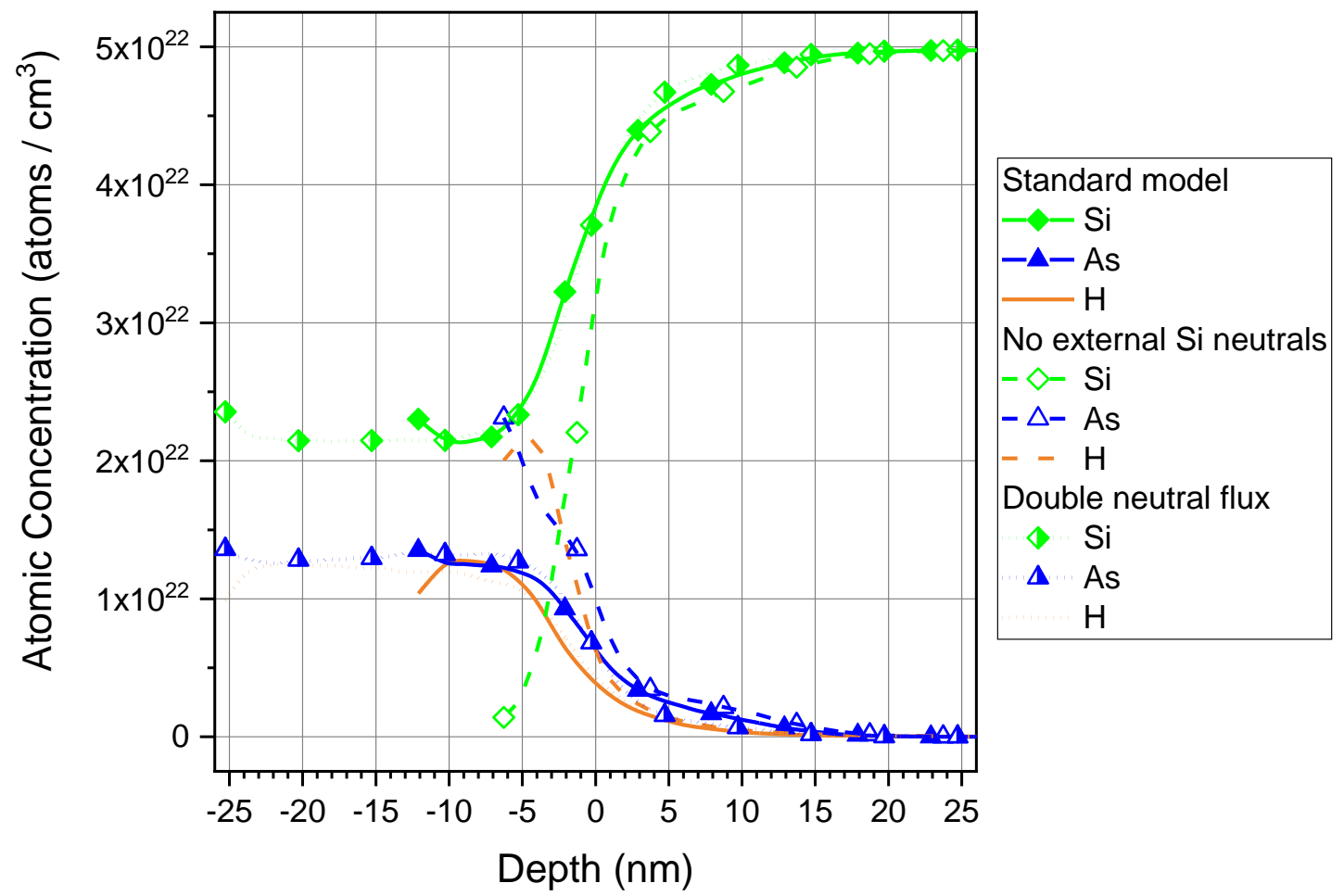

Figure 5. A comparison of TRIDYN model with constant fluxes. The origin of the depth scale is the original surface of wafer.

\section{Post Clean}

\section{TEM/EDS}

Figure 6 shows a cross sectional TEM image of a post clean sample with TEM/EDS data superimposed. The data can be interpreted that the wet clean had removed all of the As from a $12 \mathrm{~nm}$ thick surface layer down to region marked by a dark stripe in the TEM image. The Si left behind in the surface layer had been oxidised. The roughness of the interface with the Ir capping layer and reported presence of Ir in the oxide, suggests that 
the TEM lamella preparation steps had disturbed the surface of the sample.

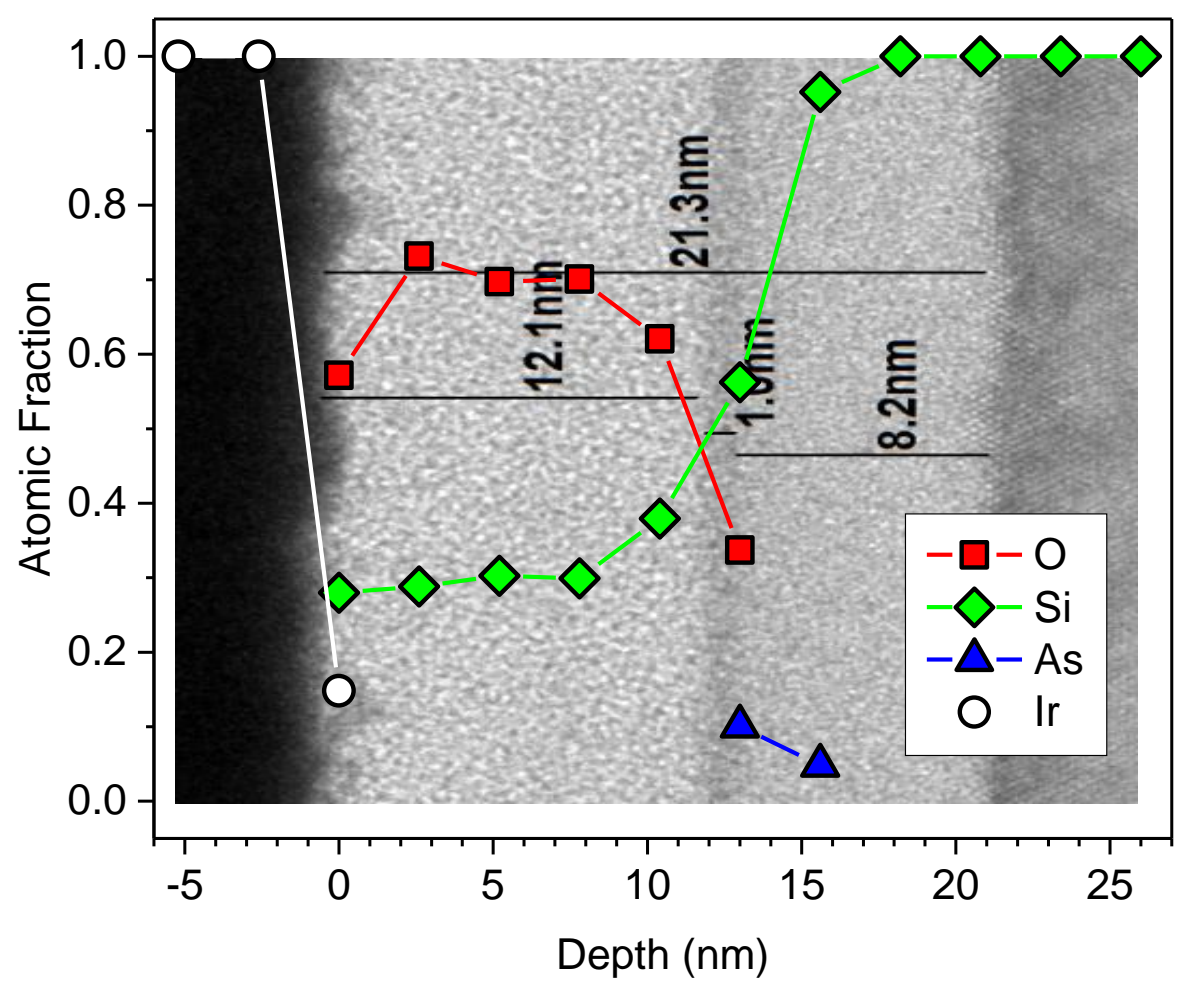

Figure 6. TEM cross section for a post SPM clean sample with EDS measurements superimposed. The origin of the depth axis is taken as bottom of iridium cap.

\section{MEIS and TRIDYN Model Predictions}

MEIS measurements (described more fully in the companion papers ${ }^{3,4}$ ) were made on a similar post wet clean sample. Figure 7 shows the elemental profiles extracted from the random and aligned orientation spectra using POWERMEIS using the as-implanted Si profile predicted by TRIDYN to guide the analysis.

The TRIDYN model for the SPM clean assumed that the as- implanted Si profile was unchanged by the clean, the as-implanted As profile was entirely removed from the 
oxide layer above the original wafer surface but was undisturbed in the bulk. It was assumed that all the $\mathrm{H}$ escaped from the oxide during the clean.

The amount of $\mathrm{Si}$ in the oxide at the surface is around $10 \%$ below the value of $2.3 \times 10^{22} \mathrm{Si}$ atoms $/ \mathrm{cm}^{3}$ expected in fully stoichiometric $\mathrm{SiO}_{2}$ but rises leading to a $\mathrm{Si}$ rich oxide towards the original surface. To indicate the sensitivity of the fit, using $\mathrm{Si}$ concentration values of $2.3 \times 10^{22} \mathrm{Si}$ atoms $/ \mathrm{cm}^{3}$ or larger throughout the layer would move the As profile outwards by $\sim 1 \mathrm{~nm}$.

A precise fit of oxygen profile is difficult because the $\mathrm{O}$ peak in the MEIS spectrum was a small signal (the ion scattering cross section decreases with target mass) superimposed onto a large signal from the abundant, Si substrate. For the oxygen profile shown in Figure 7, it was assumed that the oxygen concentration could not exceed either twice the concentration of $\mathrm{Si}$ or that of stoichiometric oxide $\left(4.6 \times 10^{22} \mathrm{O}\right.$ atoms $\left./ \mathrm{cm}^{3}\right)$. The ratio of oxygen to silicon is consistent with the ratio reported by TEM/EDS shown in Figure 6. 


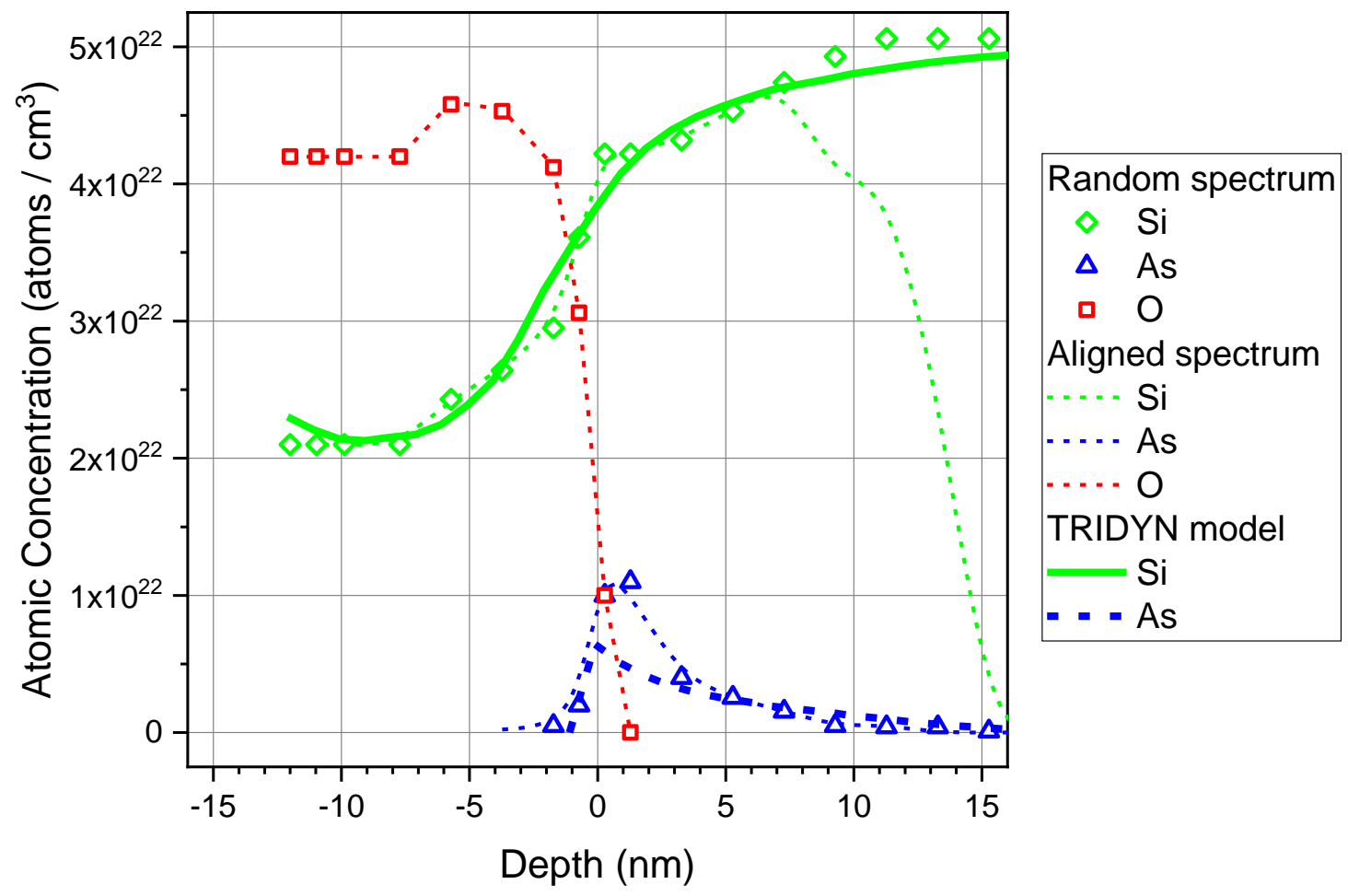

Figure 7. Elemental depth profiles extracted from aligned and random orientation MEIS measurements on a post SPM clean sample compared to TRIDYN model predictions.

Analysis of the aligned spectrum in Figure 7 suggests damage down to $\sim 16 \mathrm{~nm}$ below the original wafer surface. This is deeper than the $8-9 \mathrm{~nm}$ suggested by the amorphous/crystalline interface position in the TEM image but not as deep as the range of displaced Si atoms predicted by TRIDYN. This confirms that MEIS may be sensitive to damage, such as point defects, that are not visible in $\mathrm{TEM}^{20}$. There is also evidence for $\mathrm{H}$ damage in the post clean TEM cross sections. The $\mathrm{Si}$ and $\mathrm{O}$ profiles in the oxide for the random and aligned profiles are identical, as expected, since the oxide was amorphous. The As profiles in the aligned and random profiles are very similar, indicating that most of 
the As is interstitial, as might be expected. The SPM clean had removed almost all of the As down to the position of the original wafer surface. The As concentration there was higher than the as-implanted value and the TRIDYN values which may be a consequence of "snow ploughing" by the oxidation process. If the dark stripe in the TEM does indeed show the location of peak As concentration and the interface of the oxide and bulk silicon, this was close to the original unimplanted wafer surface.

\section{Post Anneal}

\section{TEM/EDS}

The cross sectional TEM image of a post anneal sample (Figure 1) shows that the oxide layer did not change appreciably in thickness during the anneal. TEM/EDS measurements were not made on any post anneal samples.

\section{MEIS and TRIDYN Model Predictions}

MEIS measurements ${ }^{3,4}$ were made on a similar post anneal sample. Figure 8 shows the elemental profiles extracted from the random and aligned spectra. It can be seen that the post anneal POWERMEIS Si profile was different to the TRIDYN post implant Si profile. The random Si profile suggests that the energy introduced by the anneal allowed $\mathrm{Si}$ to diffuse from the $\mathrm{Si}$ rich part of the oxide layer so that the $\mathrm{Si}$ bulk and $\mathrm{SiO}_{2}$ layers could reach their full densities. In the aligned Si profile, after accounting for scattering from the first layer of Si at the oxide interface, the reported "displaced Si" may be due to strain of the Si lattice due to the presence of interstitial As rather than residual implantation damage that had not recrystalised during the anneal. 


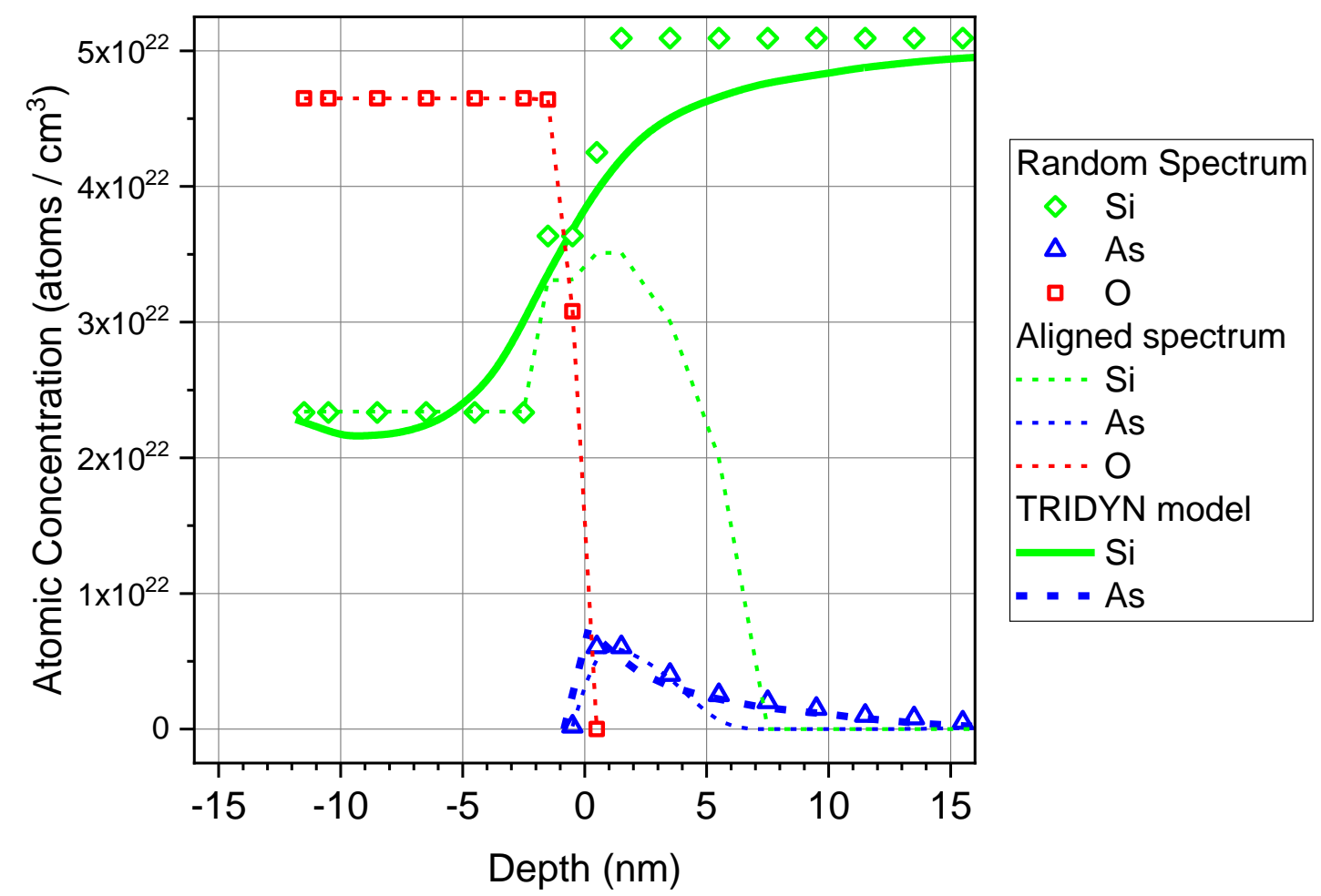

Figure 8. Elemental depth profiles extracted from aligned and random orientation MEIS measurements on a post annealed sample compared to TRIDYN model predictions.

Comparison between the As profiles in the post anneal (Figure 7) and post clean (Figure 6) random profiles suggest that As has diffused as expected during the anneal into the substrate. The total amount of As in each profile was the same $\left(3.8 \times 10^{15} / \mathrm{cm}^{2}\right)$ showing that all of the As had been retained during the anneal. The post anneal random and aligned As profiles (Figure 8) in the two profiles can be integrated and their difference (which is deeper than $4 \mathrm{~nm})$ suggests that $45 \%\left(1.7 \times 10^{15} \mathrm{As} / \mathrm{cm}^{2}\right)$ of the arsenic is substitutional, and therefore presumably activated; Steen et $\mathrm{al}^{21}$ have found that some of the "interstitial" As at the oxide interface could also be electrically active. 


\section{E. Summary of the PLAD and Post Implant Processes}

The TEM cross sectional images, EDS measurements and MEIS data suggest the PLAD implant, post clean and anneal processes progressed as follows:

During the PLAD implant neutral species containing As, Si and $\mathrm{H}$ were deposited onto the wafer surface. Si and As ions amorphised the Si substrate, mixed the deposited As and Si neutrals into the wafer bulk and mixed Si from the bulk into the deposited layer. Most of the As present in the bulk of the wafer at the end of the implant had been introduced via the ion beam mixing mechanism, rather than by the direct implantation of an ion. A large fraction of the Si in the deposited layer must have originated from the outside of the wafer, rather than from the bulk. Oxidation of the deposited layer after the implant, both by the intentional passivation treatment and subsequent exposure to atmosphere, progressed from the surface inwards, both releasing As from surface of the deposited layer and pushing some of the As inwards. The migration of As left the deposited Si atoms undisturbed from their post implant positions.

The chemical action of the SPM clean removed most of the As from the deposited layer down to the vicinity of the original wafer surface and pushed a small amount of As from the deposited layer into the Si bulk. The distribution of Si atoms left behind did not appear to change from their post implant distribution. The oxidation of this "Si scaffold" created a graded oxide layer that was $\sim 10 \%$ less dense than thermally grown oxide at the surface becoming progressively Si rich with depth. The layer around the original wafer surface, observed as the dark stripe in TEM cross section images due to "Z" and/or phasecontrast, was a mixture of As, Si and $\mathrm{O}$ and contained the highest concentration of As. 
The energy imparted to the wafer during annealing caused both As and Si atoms to diffuse. The thickness of the silicon oxide layer did not change greatly but became fully dense. The Si bulk recrystallized, and became fully dense as a result of the Si ions that had been implanted being made available to repopulate the sub surface region from which $\mathrm{Si}$ atoms had been removed. An amorphous region may have remained at the oxide interface and some lattice strain may have been present due to the presence of As in the lattice. All of the As was retained during the anneal with some inward diffusion and incorporation of $45 \%$ of the As into the lattice. A post anneal DHF was able to remove the silicon dioxide layer.

\section{Conclusions}

Analyses of a range of measurement techniques model and the consideration of elemental concentrations rather than just atomic fractions alongside a TRIDYN model has enabled valuable insights into a plasma doping and its subsequent process steps.

MEIS allowed elemental concentration depth profiles to be measured which was not possible using TEM/EDS, which could only report atomic fractions, or SIMS, whose interpretation would have been uncertain due to matrix dependency on the instantaneous sputter rates and secondary ion coefficients. However, the extraction of depth profiles from MEIS spectra does require interpretation which means that there may not always be a unique depth profile that can fit the data. It must therefore be emphasised that guiding MEIS analyses to agree with TRIDYN simulations as such does not prove anything, but has allowed the proposal of a quantified, self-consistent model to describe the mechanisms occurring during a PLAD implant, clean and anneal. 
Whilst the PLAD implant mechanisms proposed are known, this study has characterised their relative contributions at different stages of the particular PLAD implant investigated. The magnitudes of ion and neutral fluxes of the $\mathrm{Si}$, As and $\mathrm{H}$ were quantified, which in turn led to proposals for the source of the ions and neutral species. The relative importance of $\mathrm{Si}$ neutrals originating from the plasma chamber and $\mathrm{Si}$ bulk in the “deposited" layer was particularly interesting.

The TRIDYN model in itself was an oversimplification because it assumed fluxes that remained constant throughout the implant. Whilst this is known to be incorrect, there was no need within this study to extend the complexity of the TRIDYN model. Future studies of planar samples would benefit from more precise measurements of the oxygen profiles and elastic recoil detection (ERD) measurements would be valuable to measure $\mathrm{H}$ compositions in the layers, which are as yet un-known.

\section{ACKNOWLEDGMENTS}

JE would like to thank his colleagues at Varian Semiconductor Equipment Business Unit, Applied Materials for data, discussions and help in preparing this paper; in particular the Plasma Doping Group, especially Devan Raj who collected the seminal first set of data that suggested this study, but also Cuiyang Wang, Harold Persing, Nicholas Bateman and, Vikram Bhosle, Mitchell Brocklebank and Nicholas Chamberlain. Wolfhard Moeller, of HZDR, was inspirational in his collaborative work on ion beam modelling with the codes he is developing. Pedro Grande, Igor Alencar, Gabriel Marmitt and Henrique Trombini from Universidade Federal do Rio Grande do Sul, Porto Alegre, Brazil contributed useful discussions, advice and access to POWERMEIS. 
${ }^{1}$ J. England, W. Möller, J.A. van den Berg, A. Rossall, W.J. Min, J. Kim, Nucl. Instrum. Methods B 409, 60 (2017).

${ }^{2}$ E. Demenev, D. Giubertoni, J.A. van den Berg, M. Reading, M. Bersani, Nucl. Instrum. Methods B 273, 192 (2012).

${ }^{3}$ J. Van den Berg, A. Rossall, J.England, (2019) IEEE Conf Proc, in press.

${ }^{4}$ J. Van den Berg, A. Rossall, M. Hussey, J.England, J. Vac. Sci. Technol. B (2019), in press.

${ }^{5}$ W. Möller and W. Eckstein, Nucl. Instrum. Methods B 2, 814 (1984).

${ }^{6}$ M. Sortica, P. Grande, G. Machado and L. Miotti, J. Appl. Phys. 106, 114320 (2009).

${ }^{7}$ T. Miller, L. Godet, G. D. Papasouliotis and V. Singh, AIP Conf. Proc. 1066, 457 (2008).

8“Piranha solution", retrieved on $5^{\text {th }}$ October 2018 from https://en.wikipedia.org/wiki/Piranha_solution,

${ }^{9}$ Applied Materials Implant Division, Gloucester, MA USA, private communication

${ }^{10}$ J. Van den Berg, P. Bailey, R. Barlow, T. Noakes, S. Kilcoyne and R. Cywinski, 'The UK MEIS facility: a new future at the IIAA, Huddersfield' in 23rd Conference on Accelerators in Research and Industry, 25-30 May 2014, San Antonio, Texas, USA.

${ }^{11}$ A. Vantomme, Nucl. Instrum. Methods B 371, 12 (2016).

${ }^{12}$ J.F. Ziegler, M.D. Ziegler, J.P. Biersack, Nucl. Instrum. Methods B 268, 1818 (2010).

${ }^{13}$ H. Hofsass, K. Zhang, A. Mutzke, Appl. Surf. Sci. 310, 134 (2014).

14،"TRIDYN, 1D Dynamic Computer Simulation of Ion Irradiation Processes Using the Binary Collision Approximation User Guide (Version 30-Mar-2017)”, W. Möller, Institute of Ion Beam Physics and Materials Research, Helmholtz-Zentrum DresdenRossendorf 01314 Dresden, Germany, p16. 
${ }^{15}$ S. Qin, J.D. Bernstein, C. Chan, J. Shao, S. Denholm, Surf. Coat. Tech. 85, 56 (1996).

${ }^{16}$ L. Wang, R. K. Y. Fu, X. Zeng, P. K. Chub, W. Y. Cheung, S. P. Wong, J. Appl. Phys. 90, 1735 (2001).

${ }^{17}$ D. Boutard, B. Scherzer, W Moeller, J. Appl. Phys. 65, 3833 (1989).

${ }^{18}$ Korean Materials Analysis Corporation, Daejeon, South Korea, private communication.

${ }^{19}$ S. Choi, M. Numan, W. Chu, J. Srivastava, E. Irene, Appl. Phys. Lett. 50, 688 (1987).

${ }^{20}$ J. van den Berg, G. Carter, D. Armour, M. Werner, R. Goldberg, E. Collart, P. Bailey and T. Noakes, Appl. Phys. Lett. 85, 3074 (2004).

${ }^{21}$ C. Steen, A. Martinez-Limia, P. Pichler, H. Ryssel, S. Paul, W. Lerch, L. Pei, G. Duscher, F. Severac, F. Cristiano, W. Windl, J. Appl. Phys. 104, 023518 (2008). 


\section{TABLES}

Table 1. Fluxes considered in the TRIDYN model.

Type Description

$\mathrm{As}^{+} \quad$ Generated from the plasma feed gas and from material sputtered from the wafer or chamber walls (either as charged particles or as neutrals which are subsequently ionised in the plasma). Multiply charged ions, As dimers and $\mathrm{H}$ associated with the charged As species have not been included in the TRIDYN model.

$\mathrm{Si}^{+} \quad$ These can be formed by ionisation in the plasma of Si sputtered from the wafer and chamber walls or liberated by chemical etching of quartz or $\mathrm{SiO}_{2}$ layers by $\mathrm{H}$ radicals. This flux is required in the TRIDYN model.

$\mathrm{H}+\quad \mathrm{H}$ monomer, dimer and trimer peaks are routinely observed by residual gas analysers ${ }^{10}$. Energetic hydrogen ions formed from the $\mathrm{H}$ feed gas and even $\mathrm{H}$ introduced as part of $\mathrm{AsH}_{\mathrm{x}}{ }^{+}$ions deeply penetrate the sample beyond the region of interest in this study. For this reason $\mathrm{H}+$ ions have not been included in the TRIDYN model.

$\mathrm{As}^{0} \quad$ Generated from the plasma feed gas and from material sputtered from the wafer or chamber. Some of these neutrals represent $\mathrm{AsH}_{\mathrm{x}}$ molecules that contain arsenic and one or more hydrogen atoms.

$\mathrm{Si}^{0} \quad$ Sputtered from the wafer and the chamber. 
$\mathrm{H}^{0} \quad$ Unlike the ions, hydrogen introduced as members of $\mathrm{AsH}_{\mathrm{x}}$ molecules will remain in the deposited layer of interest for this study. The volume taken up by this $\mathrm{H}$ will account for the As not taking up the full density of elemental As. 


\section{Figure Captions}

Figure 1. TEM cross sections of samples from different process steps a) several days after implant; b) after an SPM clean; c) post anneal; d) after DHF oxide removal. All the cross sections are shown at the same scale.

Figure 2. TEM cross section of a post implant sample with EDS measurements superimposed. The origin for the depth axis taken as the bottom of the iridium cap.

Figure 3. Elemental profiles (symbols) for the post implanted sample extracted from the post implant MEIS spectra using POWERMEIS. The lines show predictions for the $\mathrm{Si}, \mathrm{As}$ and $\mathrm{H}$ profiles from the TRIDYN model. The oxygen was introduced after the implant and is not part of the TRIDYN model; $\mathrm{H}$ cannot be measured by MEIS. The origin for the depth axis has been taken as the original surface of the wafer prior to implantation in the TRIDYN model.

Figure 4. Elemental depth profiles and the contributions from ion and neutral species for the post implanted sample predicted by the TRIDYN model. The damage to the bulk Si is expressed as displacements per atom.

Figure 5. A comparison of TRIDYN model with constant fluxes. The origin of the depth scale is the original surface of wafer.

Figure 6. TEM cross section for a post SPM clean sample with EDS measurements superimposed. The origin of the depth axis is taken as bottom of iridium cap.

Figure 7. Elemental depth profiles extracted from aligned and random orientation MEIS measurements on a post SPM clean sample compared to TRIDYN model predictions.

Figure 8. Elemental depth profiles extracted from aligned and random orientation MEIS measurements on a post annealed sample compared to TRIDYN model predictions. 